\title{
Research into Various Aspects of Loss Brought by Urban Traffic Congestion and Countermeasures
}

\author{
Zixin Song ${ }^{1} \mathrm{a}^{*}$, Liyi Tian ${ }^{1}$, Chao Zhang ${ }^{1}$, Yucong Wu${ }^{1}$ and Qian Luo ${ }^{1}$ \\ ${ }^{1}$ No.2 Beinong Road, Huilongguan Town, Changping District, Beijing, North China Electric Power \\ University
}

a297974540@qq.com

Keywords: Traffic jams; Huilongguan; Loss; Recommendations

\begin{abstract}
With the development of economy, Chinese urbanization has accelerated already, the people's living standards and quality of life have also increased steadily, and car ownership has gradually increased. Traffic congestion caused by a series of reasons including road planning and traffic control has become a big problem which cannot be ignored blocking the urbanization city. This paper chooses Huilongguan area as the research object, visiting the residents there and field research, we acquire the relevant data and then analyze environmental pollution, energy consumption and various losses which brought by the traffic congestion. Finally, provide the improvement of the relevant recommendations.
\end{abstract}

\section{Introduction}

Concept of Congestion. For traffic congestion, the Texas Department of transportation proposed the definition of traffic congestion: when the travel time more than in small traffic flow or free flow travel environment under normal incidence of travel time, produce larger delay of traffic state, when the delay exceeds the public to generally accepted boundaries, said unacceptable traffic congestion. [1]

Research Background. In the past 20 years, our country has obtained the unprecedented development. However, the traffic jam problem in some cities is also unprecedented. According to the website of the National Bureau of Statistics announced the 2014 National Economy and Social Development Statistical Bulletin, by the end of 2014, national civilian car retains the quantity to 15447 million vehicles, up 12.4 percent over the previous year, which private car retains the quantity 125.84 million, an increase of $15.5 \%$. Civilian car ownership 83 million 70 thousand, a growth of $16.6 \%$, of which 75 million 900 thousand private cars, up 18.4\%. With the improvement of the quality of life, the public has a higher demand for their own travel quality, and then the rapid increase in private car ownership. While a huge number of floating population and the increasing pressure of life, strength of the production and living of the city increased, leading to a sharp rise in the demand for motor vehicles, the whole city's traffic pressure continued to increase; on the other hand, the traditional road traffic facilities have been unable to meet the needs of modern society, and in the construction of urban roads by urban investment and use restrictions, resulting in new and expansion of urban road slow progress, urban traffic supply capacity is insufficient, serious impact on the quality in the daily life of the people and the city's sustainable development.

Current Traffic Congestion in China. According to data released by the Ministry of Public Security Traffic Management Bureau, among 667 cities nationwide, about two thirds of the urban traffic in rush hour congestion. These data show that on the one hand, China's car ownership rose in a very large proportion of; and on the other hand, China's many large cities traffic congestion problem is quite serious, has become the norm and have exacerbated the trend, some second and third tier cities is rapidly entering the congestion times ". With the car's rapid growth and urbanization speeds up, expand the scale of China's overall congestion, increasing road congestion, congestion is becoming more and more serious, vehicle speed is more and more slow, congestion time more and longer. At present, the 32 million more than the population of big city, road area per capita in 27 cities has been lower than the national average level; in the late 1990s, lane of Shanghai 
urban centers such as the $50 \%$ peak hour saturation is reached $95 \%$, saturation throughout the day more than $70 \%$, the average speed dropped to $10 \mathrm{~km} / \mathrm{h}$. Traffic jam seriously affects people's travel and life.

Significance of Research. (1) Predict loss to provide theoretical basis for improving traffic.(2) Improve the environment and residents' life quality.(3) Alleviate traffic congestion to provide guarantee for urbanization.

\section{Investigation}

Investigation Process. (1) Collect Information: Go to the library and read books and literature and in the online search related information, to understand the Huilongguan area congestion points, peak congestion and the current congestion, including news reports, journals, and video data.

In the Huilongguan community, ask the local residents to travel, travel time, travel to the whereabouts of the traffic congestion and make the basic situation of the corresponding statistics.

According to the relevant information has been learned, choose the peak of the morning and evening peak congestion points, the number of vehicles and the number of vehicles and the retention time and other statistical data.

(2) Information Process: Summarize, classify, gather and analyze the inquired books and a huge amount of materials obtained from the Internet. Discuss them to get some preliminary conclusions. Collect the data obtained from investigation and analyze with statistical knowledge and then male a diagram to show the conclusion in a more intuitive way.

(3) Logic Analysis: Combine the results with relevant materials to establish suitable models to predict the loss and propose suggestion to improve traffic congestion.

(4) Communication, Summary and Thesis Writing: Discuss and communicate with all members and write the formal thesis on the basis of fully understanding the above investigation results and conclusion.

Basic Situation of Investigation. Huilongguan is located in the south of Changping District, Beijing, with a total population of about 450 thousand people, with an area of $34.5 \mathrm{~km} 2$. As one of the largest residential areas in Beijing City, the traffic problem has been a major problem plagued the residents of the area; the region is also one of the main traffic jam in Beijing city. Huilongguan, Huoying, and added larger residential area near Huilongguan area of distribution, population and more, "post separation" phenomenon, resulting in traffic in the area appear tide phenomenon. Work on the morning and evening peak time especially congestion

\section{A: Visit Residents}

\section{(1)Travelling Way}

According to the results of the survey, Huilongguan residents travel mainly by three means, respectively private cars, buses and subway, respectively, accounting for $36.5 \%$ of the total travel, $30.5 \%, 21.5 \%$, including bus, another way to travel for travel $11.5 \%$ of the total.

(2) Travelling Time

Residents' travel time in the morning is mainly concentrated in 06:00-08:00, accounting for 84.5\% of the total, starting at the time mainly concentrated in 17:00-19:00, accounting for $81.5 \%$ of the total. The evening arrived in Huilongguan time to focus on 18:00-20:00, accounting for 83\% of the total.

(3) Travelling Direction

According to the project group on the characteristics of Huilongguan area residents travel survey shows that, the Huilongguan area residents work (Study) traffic to attract a large amount of area followed by the Haidian District, Chaoyang District, Changping District, Dongcheng District, Xicheng District and Huilongguan area in five regions, respectively, accounted for $40.5 \%$ of the total proportion of travel, $16.5 \%, 15.0 \%, 14.5 \%$ and $14.0 \%$. According to the survey, Huilongguan area residents gathered mainly distributed in Shangdi and Zhongguancun, Huilongguan, Qinghe, ITC, and Xidan.

(4) Opinions of Traffic Congestion

It can be learned from the talk with the residents that most of the residents think that the current 
traffic has greatly satisfied their demand for transportation. At the same time, however, due to the many vehicles and intense population in many aspects, traffic congestion of people caused great distress. $81.5 \%$ of residents believe that traffic conditions need to be improved, $11.5 \%$ of residents said it does not matter, $7 \%$ of residents believe that the current traffic congestion situation did not cause trouble for travel.

B: Field Investigation

(1) Xinzhuang Bridge

Xinzhuang Bridge is located at the intersection of Badaling Highway, Changping District, Beijing and Beijing Road. Here the road is complex, Beiqing Road and S216 Road intersection, a wide range of vehicles.

In the late peak period, a red light period, an average of about 350 vehicles on the side of the road, which accounted for $77.14 \%$ of private cars, bus $4.57 \%$, taxi accounted for $3.14 \%$, including trucks, including other vehicles accounted for $15.15 \%$. The red light green light after a period of about $90 \%$ vehicles can pass the Xinzhuang bridge.

(2) Longze Railway Station

Longze subway station is a station of Beijing subway line 13, and it is located to the southeast of the cross of G6 Beijing-Tibet highway and Tongcheng Street.

Longze subway station is surrounded by large residential communities like Longzeyuan, Longhuayuan and Fengyayuan without other subway stations in the surroundings. Therefore, Longze subway station has become the first choice for nearby residents to take the subway. According to investigation, at the morning peak hours, every one spends 20 minutes to enter the subway station.

\section{Prediction of Loss}

Consumption of Fuels. The cost of additional fuel consumption refers to the additional cost of fuel oil, which is caused by the increase in fuel consumption due to congestion in urban roads. This cost and congestion peak speed, average fuel efficiency of each type of vehicle, fuel prices and other variables. As the city's vehicle fuel contains three kinds of gasoline, diesel and gas, the cost of fuel consumption should also be divided into three categories to calculate. In view of the traffic management measures in Beijing City, the traffic congestion occurs almost all buses, and most of the use of gasoline, so this paper takes the maximum consumption of gasoline as an example to calculate the formula for:

Extra daily fuel consumption $=$ daily delay at peak hours $\times$ average fuel efficiency at traffic congestion $\times$ fuel price

Car delay time at peak hours = delay time of various types of car at each section $\times$ the number of every car at each section

Average daily delay time for each section of the road to the vehicle through the congested sections of the average time used to subtract the average time when the road is smooth [3].

Average fuel efficiency refers to the number of liters of gasoline consumed per unit of time. Due to the congestion caused by the engine does not stop when parking, this idle stop and no kilometers, so consider the time to measure the amount of fuel consumption. According to the test, the idling stop is 3 minutes is equivalent to $1 \mathrm{~km}$, to every car average fuel consumption per 100 kilometers 8 liters to calculate, is every 3 minutes for parking congestion produced fuel consumption is 0.08 liters, fuel consumption per minute for parking congestion produced about $0.027 \mathrm{~L}[4]$.

In fact, frequent start-stop idling stop will further aggravate fuel consumption. Through testing, an emergency stop and rapid start, it will consume 35 milliliters of gasoline; rapid start 10 times, increase fuel consumption $120 \mathrm{ml}$ or more; empty throttle 10 times, wasting fuel more than $60 \mathrm{ml}$; sudden acceleration than the smooth acceleration to consume 1/3 of the fuel. In addition, congestion will lead to increased use of time vehicles, which no doubt increases the fuel consumption. Beijing is calculated daily additional fuel loss of about 55,086,200 yuan, the year due to traffic congestion caused by the additional fuel consumption of 20.106 billion yuan [5]. 
Environmental Pollution. In the urban environment, motor vehicle pollution emission is one of the main causes of air pollution. Motor vehicle pollution emissions include: particulate matter, hydrocarbons (HC), sulfur dioxide (SO2), nitrogen oxides (NOX) and carbon monoxide (CO), etc. Domestic and foreign research shows that urban road traffic, when the speed to maintain in the $15 \mathrm{~km} / \mathrm{h}$, emissions of carbon and oxygen compounds is speed $35 \mathrm{~km} / 1.55$ times [6], while at the same time carbon monoxide emissions up to 1.9 times, and if the vehicle is in the idle state, vehicle emissions of pollutants will be higher.

Residents' Health. Road traffic congestion will cause a certain impact on the health of the public.

On the one hand, road traffic congestion will affect the mood of drivers and passengers, resulting in mental illness. In the crowded roads both drivers or passengers are relatively easy to form anxiety, depressed mood, for heart patients, traffic congestion will increase heart load, easy to induce heart disease. If the long-term in the mood and cannot adjust the normal to will work will affect the normal production, brought to life will affect the individual's physical and mental health, caused by mental illness; at the same time in the mood under, the driver's patience will greatly reduce, easy to produce fatigue driving and more easily triggered traffic accident. On the other hand, road traffic congestion will affect the health of drivers, passengers and nearby residents. As the traffic jam caused by the increase of pollutant emissions, the driver and passengers as well as the surrounding residents to breathe more toxic and harmful gases, causing damage to their bodies[7].

\section{Measures for Improvement}

Implement Road Planning. Aiming at the traffic congestion at Huilongguan area caused by dead end roads, this issue can be resolved by planning roads again [8]. The specific measures are:

(1) Break dead end road to realize connection of the south and the north

(2) Construct roads north to Taiping Village to enhance connection between the east and the west.

(3) Construct eastern roads and enhance lengthways connection

(4) Strength connection between Huilongguan area and Beijing-Baotou highway

Optimize Public Transportation. According to a rough estimation, is a car keeps driving on a lane, it can only transport about 2,000 people in an hour while the bus traveling at the same time can transport thousands of people. Single car on patronage, the one-way bus passenger capacity is 100 times that of the bicycle, 30-40 times sedans. So, in addition to rail transport, the bus is better than other modes of transport, is one of the core elements of bus priority development strategy [9].

Promote Car-Pooling. As a new type of quick and convenient means of traffic, car-pooling is the first choice for low-carbon travelling, and it has developed mature abroad, but it enjoys low popularity in China. Instead, there are overcrowded roads and railways.

Nowadays, some domestic advanced car sharing software can bring people's usually scattered disorderly carpooling demand into a management platform, while landing the PC web site, Ann Zhuo mobile client and apple mobile client platform, in the private car link, drivers and passengers through the platform of a, registration, login, release and recharge four main operation. If the carpool way can be promoted in the Huilongguan area, the traffic pressure will be greatly reduced [10].

\section{References}

[1] Wang Dong, Research into Automatically Identify Urban Traffic Congestion: (graduate dissertation). Guizhou: Guizhou University, 2008.

[2] Li Lin, Research into Predicting Costs of China's Urban Traffic Congestion and Countermeasures: (graduate dissertation). Dalian: Dalian Maritime University, 2013.

[3] Wu Dongdong, Evaluation of Ecological Economic Loss Brought by Traffic Congestion in Beijing, Ecological Economy, 2013(4): 75-79 
[4] Feng Xiangzhao, Zou Ji, Guo Guangming. Evaluation of External Costs of Urban Traffic Congestion [J]. Environment and Sustainable Development, 2009(3): 1 4.

[5] Luo Qingyu, Juan Zhicai, Sun Baofeng, etc. Research into Methods to Measure External Costs of Urban Traffic Congestion [J]. Transportation System Engineering and Information, 2007(7): $9 \sim 12$.

[6] Jin Lili. Analysis of External Costs of Urban Transportation and Internal Quantitative Method [J]. Traffic Science and Economy, 2007(9): 103 105.

[7] Guo Ruijun, Wang Wanxiang. Preliminary Analysis of Urban Traffic Congestion's Social Costs [J]. Urban Public Transportation, 2008(4):26 29.

[8] Lin Jinkun, Analysis of Causes of Traffic Congestion at Huilongguan Area in Beijing and Measures for Improvement [B]. Road and Traffic Engineering, 2015(3).

[9] Zhang Dexin, Xv Ke, Han Jun, Cao Xintao. Research into Causes of Traffic Congestion at Huilongguan Area and Countermeasures [B]. Traffic Engineering, 2014(12).

[10]Sun Wenjing. Research into Urban Traffic Congestion from the Perspective of Coordinated Treatment: (graduate dissertation). Nanning: Guangxi University. 